\title{
YpeB dimerization may be required to stabilize SleB for effective germination of Bacillus anthracis spores
}

\author{
Cameron V. Sayer and David L. Popham ${ }^{*}$
}

\begin{abstract}
Background: Bacillus cells faced with unfavorable environmental conditions undergo an asymmetric division process ultimately leading to the formation of the bacterial spore. In some instances the spore serves as an infectious agent; such is the case with the spore of Bacillus anthracis and the disease anthrax. Spores are resistant to a variety of environment conditions including traditional decontamination techniques due to the formation of specialized cellular structures. One such structure, the spore cortex, is a thick layer of modified peptidoglycan that contributes to spore dormancy through maintenance of the dehydrated state of the spore core. During spore germination, degradation of the cortex is required to facilitate complete hydration of the core and a return to vegetative growth. Degradation of the cortex is accomplished through the action of germination-specific lytic enzymes. One of these enzymes, SleB, has been previously shown to require the presence of the YpeB protein for its stable incorporation and subsequent function in spores of $B$. anthracis. The focus of the present study is to identify protein interactions of YpeB through in vivo chemical cross-linking and two-hybrid analysis.

Results: Conserved residues within YpeB PepSY domains were altered to facilitate implementation of a site-specific chemical cross-linker, 4-Azidophenacyl bromide. Analyses of crosslinked-spore extracts suggests that YpeB exists as a dimer or larger multimer within the spore, potentially mediated through interactions of the C-terminal domains. Spores expressing stable truncated forms of YpeB were crosslinked and corresponding truncated dimers were detected. Further characterization of individual YpeB domains using bacterial two-hybrid analysis indicated a possible role for both $\mathrm{N}$-and $\mathrm{C}$-terminal domains in YpeB oligomerization.

Conclusions: The YpeB protein likely exists as dimer or higher-order multimer in the dormant spore. Both the Nand C-terminal YpeB domains contribute to multimerization. SleB likely also exists as an oligomer, and SleB and YpeB may be found together within a protein complex. Disassembly of this complex during spore germination likely allows SleB to become active in spore cortex degradation. Further study of this protein complex may contribute to the development of methods to inhibit or stimulate germination, allowing more effective spore decontamination.
\end{abstract}

Keywords: Bacillus, Endospore, Spore, Germination, Cortex

\footnotetext{
* Correspondence: dpopham@vt.edu

Department of Biological Sciences, Virginia Tech, 926 West Campus Drive,

Blacksburg, VA 24061, USA
}

(c) The Author(s). 2019 Open Access This article is distributed under the terms of the Creative Commons Attribution 4.0 International License (http://creativecommons.org/licenses/by/4.0/), which permits unrestricted use, distribution, and reproduction in any medium, provided you give appropriate credit to the original author(s) and the source, provide a link to the Creative Commons license, and indicate if changes were made. The Creative Commons Public Domain Dedication waiver (http://creativecommons.org/publicdomain/zero/1.0/) applies to the data made available in this article, unless otherwise stated. 


\section{Background}

Bacillus anthracis has the potential to cause widespread illness and severe disease through multiple routes of infection. As with many other disease-causing endosporeproducing bacterial species, the bacterial endospore serves as the infectious agent of the disease anthrax [1]. This is especially problematic because the inherent resistance characteristics of bacterial spores render many standard decontamination methods ineffective [1-3]. The greatest factor in maintenance of spore resistance properties is preservation of the metabolically dormant and dehydrated state of the spore core [4]. Dormancy is maintained by specialized spore structures including the inner spore membrane and cortex peptidoglycan, and high spore core concentrations of $\mathrm{Ca}^{2+}$-dipicolinic acid (DPA). [2, 3, 5]. These factors contribute to the overall threat that $B$. anthracis poses, especially as a bioterrorism agent.

When the dormant spore senses an environment with favorable nutrient availability, such as within a host, it will rapidly germinate, returning to a vegetative growth state. Germination is initiated following sensing of germinants by receptors at the inner spore membrane, after which large stores of $\mathrm{Ca}^{2+}$-DPA are released from the spore core and partial rehydration of the core begins [2]. The spore cortex is then depolymerized, facilitating complete hydration of the core and a return to a vegetative growth state [2]. Completion of germination of $B$. anthracis within the host is required for production of the anthrax toxins and ultimately progression of the disease [1].

The cortex is degraded by germination specific lytic enzymes (GSLEs). B. anthracis encodes four of these enzymes, but the majority of the cortex degradation has been demonstrated to be completed through the action of partially redundant enzymes SleB and CwlJ1 $[6,7]$. These enzymes specifically recognize modified muramic$\delta$-lactam [8-13], which is found uniquely in spore cortex peptidoglycan [14, 15]. CwlJ1 is localized to the spore coat layer and has been shown to be activated by the release of $\mathrm{Ca}^{2+}$-DPA $[2,16,17]$.

In Bacillus species, both SleB and YpeB are expressed from a conserved operon and this is also true of several Clostridium strains [18]. Previous studies have determined that SleB and YpeB co-localize to the inner spore membrane as well as potentially to a second location near the outside of the cortex $[17,19]$. Further studies have determined that $\mathrm{SleB}$ and $\mathrm{YpeB}$ are co-dependent, requiring one another for stable incorporation within the dormant spore in both B. subtilis and B. anthracis [17, 20-22]. SleB and YpeB are expressed under the control of $\sigma^{\mathrm{G}}$ and are translocated across the inner spore membrane via N-terminal signal sequences $[9,19,22,23]$. The signal sequence of $\mathrm{YpeB}$ is not predicted to be cleaved, leaving YpeB anchored to the inner spore membrane, while SleB is expressed in its mature form, with signal sequence removed, within the dormant spore [19, 23-25]. Given the co-localization, co-dependency, and that SleB is present but held inactive in the dormant spore, it has been theorized that YpeB and SleB interact in some manner to stabilize one another within the dormant spore [20]. Previous studies have implicated a role for both $\mathrm{N}$-terminal (residues 21-202) and C-terminal (residues 203-446) regions of YpeB in interactions with SleB [20, 21]. It has been demonstrated that the $\mathrm{N}$-terminal domain of YpeB was most effective in inhibiting SleB activity in vitro [21], while a region of $\mathrm{YpeB}$ beyond the first PepSY domain is required for $\mathrm{SleB}$ incorporation within the dormant spore [20].

The goal of the current study was to further characterize the relationship between YpeB and SleB within the dormant spore. In vivo peptide cross-linking was used to study potential interactions of YpeB, identifying interactions that may form only within the unique environment of the dormant spore. Bacterial two-hybrid analysis was used to detect domain-specific interactions. Both methods indicate YpeB oligomer formation, which may be required for stable incorporation of SleB and subsequent germination of the Bacillus spore.

\section{Results}

In vivo site-directed cross-linking of YpeB in B. anthracis dormant spores

Previous work has highlighted the importance of the YpeB C-terminal domain (203-446), specifically residues beyond the first PepSY domain, for stabilization of SleB in the developing spore [20]. Interactions of the YpeB Cterminal domains within the dormant spore were further characterized by employing in vivo amino acid-specific chemical cross-linking. Guided by homology modeling of the YpeB C-terminal domain to that of the metalloprotease Vibriolysin [26], several amino acid residues were selected as potential interaction sites. Residues were chosen based on the following criteria: predicted to be surface exposed, critical in PepSY domain interactions in Vibriolysin, and/or conserved in ypeB orthologs. Each of the selected residues was then mutagenized, substituting the wildtype codon with that for cysteine, and YpeB-cysteine alleles were recombined into the chromosome of a $\triangle y p e B$ strain. These alleles also carried a C-terminal hexa-histidine tag for protein purification purposes. This tag has previously been shown to not interfere with YpeB function [20]. The functionality of the YpeB-Cys proteins were verified by examination of the abundance of YpeB and SleB in the dormant spores, quantification of optical density (OD) loss during spore germination, and observation of YpeB proteolysis to stable C-terminal products during germination. All 
YpeB-Cys alleles were very similar to the wild type (WT) in all of these assays. Specifically, the two alleles utilized for further studies, ypeBS358C-6His and ypeBK437C$6 \mathrm{His}$ were nearly identical to the wild type in these regards (Additional file 1). Interestingly, these two YpeBCys alleles allowed normal OD loss during germination but resulted in slow spore outgrowth, potentially indicating an additional YpeB effect on germination.

Sulfhydryl specific cross-linking was conducted using the cross-linker p-azidophencyl bromide (APB), which is a heterobifunctional cross-linker with a sulfhydryl specific $\alpha$-bromo-ketone motif in addition to a non-specific photoactivatable azide, separated by a spacer arm of $9 \AA$. In total, 12 YpeB-Cys allele-carrying strains, corresponding to 12 different amino acid substitutions across the C-terminal PepSY domains (Table 1), were created and tested in site-specific cross-linking schemes of dormant spores. Of the 12 alleles tested, those encoding Cys substitutions for residues 358 and 437 revealed higher migrating complexes of roughly $\sim 100 \mathrm{kDa}$ in anti-YpeB western blots of dormant whole spore extracts following APB cross-linking (Fig. 1). It was theorized that because these higher migrating bands appeared at roughly double the mass of the YpeB monomer $(\sim 50 \mathrm{kDa})$ that these complexes might contain a cross-linked YpeB dimer. Only a small percentage of the YpeB molecules became cross-linked, presumably due to a low efficiency of cross-linker modification of the YpeB-Cys residues (amid competition by native Cys residues in the spore coats) and potentially low efficiency of the non-specific crosslinking by the photoactivatable azide. Western blots using anti-SleB antibodies revealed no bands representing potential cross-linked YpeB-SleB complexes.

\section{In vivo site directed cross-linking of $Y_{p e B_{\Delta 25-203}}$ dormant spores}

Attempting to the further demonstrate the possibility of a YpeB dimer, similar cross-linking experiments were performed using an allele of $y p e B, y p e B_{\Delta 25-203}$, that is internally truncated within the $\mathrm{N}$-terminal domain, and that was previously demonstrated to produce a protein that was stably incorporated into the dormant spore [20]. Cys residues that were reactive in the previous assay were created in $\mathrm{YpeB}_{\triangle 25-203}$, and the alleles were recombined into both $\triangle y p e B$ and WT B. anthracis backgrounds. Dormant spores from $\mathrm{YpeB}_{\triangle 25-203}$-Cys strains were then cross-linked with APB and proteins were extracted and visualized via western blot (Fig. 2). Crosslinked extracts of $\mathrm{YpeB}_{\triangle 25-203} \mathrm{~K} 437 \mathrm{C}$ in a $\Delta y p e B$ background feature both the truncated monomer $(\sim 30 \mathrm{kDa})$ and what appears to be a truncated YpeB dimer $(\sim 60$ $\mathrm{kDa})$. Extracts of $\mathrm{YpeB}_{\triangle 25-203} \mathrm{~K} 437 \mathrm{C}$ in WT background suggest the possibility of a $\mathrm{YpeB}_{\triangle 25-203}$-WT YpeB heterodimer $(\sim 80 \mathrm{kDa})$ in addition to complexes previously visualized. We next sought to confirm that this newly visualized band contained the $6 \mathrm{x}$-His-tagged YpeB. Spores encoding $\mathrm{YpeB}_{\Delta 25-203} \mathrm{~K} 437 \mathrm{C}$ were cross-linked, and 6xHis-tagged proteins in extracts were concentrated using a $\mathrm{Ni}^{2+}$ NTA affinity column. Column elutions were visualized with western blotting (Fig. 3). The higher-migrating band $(\sim 60 \mathrm{kDa})$ was visualized in the cross-linked samples but was not seen in the uncross-linked controls, indicating the potential for multimerization of the YpeB C-terminal domain even in the absence of most of the $\mathrm{N}$-terminal domain.

\section{Analyzing individual YpeB domain contributions to multimerization using bacterial two-hybrid analysis}

A bacterial two-hybrid system was implemented to better elucidate contributions of individual YpeB domains to possible multimer formation. Individual YpeB N$\left(\mathrm{YpeB}^{\mathrm{N}} 21-202\right)$ and $\mathrm{C}$-terminal domains $\left(\mathrm{YpeB}^{\mathrm{C}} 203-\right.$ 446), full-length YpeB (lacking its signal peptide) (YpeBFull 21-446), and the SleB C-terminal catalytic domain (SleB $\left.{ }^{\mathrm{Cat}} 125-253\right)$ [28] were cloned in both pKT25 and pUT18C creating N-terminal fusions to the two domains of adenylate cyclase. Constructs were co-transformed into E. coli, which was plated on MacConkey agar supplemented with maltose, where positive domain interactions were visualized by red colony coloration. In agreement with cross-linking results, bacterial two-hybrid assays indicated that $\mathrm{YpeB}^{\text {Full }}$ self-associated. (Fig. 4). Additionally, $\mathrm{YpeB}^{\mathrm{N}}$ also demonstrated self-association while $\mathrm{YpeB}^{\mathrm{C}}$ did not. However, both $\mathrm{YpeB}^{\mathrm{N}}$ and $\mathrm{YpeB}^{\mathrm{C}}$ appear to interact with $\mathrm{YpeB}^{\text {Full }}$ indicating that both of these domains are involved in dimer or higher multimer structure formation. $\mathrm{YpeB}^{\mathrm{C}}$ interacted with $\mathrm{YpeB}^{\mathrm{Full}}$ in both orientations, while $\mathrm{YpeB}^{\mathrm{N}}$ interacted with $\mathrm{YpeB}^{\text {Full }}$ in only one orientation of the adenylate cyclase domains. This negative result might result from this specific interaction of YpeB domains placing the fusion domains too far apart for a productive interaction. Interestingly, one orientation of the fusion domains also indicates an interaction between the $\mathrm{YpeB}^{\mathrm{N}}$ and $\mathrm{YpeB}^{\mathrm{C}}$ domains. Also of note, none of the YpeB constructs tested in the bacterial two-hybrid system appeared to interact with $\mathrm{SleB}^{\mathrm{Cat}}$, although SleB ${ }^{\text {Cat }}$ did appear to associate with itself, suggesting that SleB also exists as a multimer.

\section{Discussion}

This study further characterized interactions of YpeB within the dormant spore through in vivo chemical cross-linking and the roles of individual domains of YpeB in potential multimer formation using bacterial adenylate cyclase two-hybrid assays. Both in vivo crosslinking and two-hybrid analyses indicate that YpeB forms a dimer or higher-order multimer. The $\mathrm{YpeB}^{\mathrm{N}}$ domain alone exhibits strong self-association, while the $\mathrm{YpeB}^{\mathrm{C}}$ 
Table 1 Bacterial strains and plasmids

\begin{tabular}{|c|c|c|c|}
\hline Strain & Genotype & Construction $^{a}$ & Source \\
\hline \multicolumn{4}{|l|}{ B. anthracis } \\
\hline Sterne 34F2 & $\mathrm{pXO} 1^{+} \mathrm{pXO} 2^{-}$ & & P. Hanna \\
\hline DPBa89 & $\triangle y p e B$ & pDPV392 > 34F2 & [20] \\
\hline DPBa127 & АypeB::pDPV424 (YpeB $\left.1-446-\mathrm{His}_{6} \mathrm{Er}^{\mathrm{R}}\right)$ & pDPV424 > DPBa89 & {$[20]$} \\
\hline DPBa204 & АypeB:::pDPV476 (YpeB $\left.{ }^{\mathrm{K} 437 \mathrm{C}}-\mathrm{His}_{6} \mathrm{Er}^{\mathrm{R}}\right)$ & pDPV476 > DPBa89 & This study \\
\hline DPBa205 & АypeB::pDPV477 (YpeB $\left.{ }^{\mathrm{S} 358 \mathrm{C}}-\mathrm{His}_{6} \mathrm{Er}^{\mathrm{R}}\right)$ & pDPV477 > DPBa89 & This study \\
\hline DPBa210 & АypeB::pDPV478 (YpeB $\left.{ }^{T 328 C}-\mathrm{His}_{6} \mathrm{Er}^{\mathrm{R}}\right)$ & pDPV478 > DPBa89 & This study \\
\hline DPBa211 & АyреB::pDPV479 (YpeB $\left.{ }^{\mathrm{M} 282 \mathrm{C}}{ }^{-} \mathrm{His}_{6} \mathrm{Er}^{\mathrm{R}}\right)$ & pDPV479 > DPBa89 & This study \\
\hline DPBa212 & 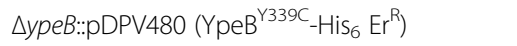 & pDPV480 > DPBa89 & This study \\
\hline DPBa213 & 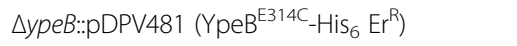 & pDPV481 > DPBa89 & This study \\
\hline DPBa214 & АypeB::pDPV482 (YpeB $\left.{ }^{\mathrm{V} 355}-\mathrm{His}_{6} \mathrm{Er}^{\mathrm{R}}\right)$ & pDPV482 > DPBa89 & This study \\
\hline DPBa215 & АypeB::pDPV483 (YpeB $\left.{ }^{\mathrm{V} 324 \mathrm{C}_{-}} \mathrm{His}_{6} \mathrm{Er}^{\mathrm{R}}\right)$ & pDPV483 > DPBa89 & This study \\
\hline DPBa216 & sypeB::pDPV484 (YpeB $\left.{ }^{T 420 C}-\mathrm{His}_{6} \mathrm{Er}^{\mathrm{R}}\right)$ & pDPV484 > DPBa89 & This study \\
\hline DPBa217 & АypeB::pDPV485 (YpeB $\left.{ }^{\mathrm{A} 342 \mathrm{C}}-\mathrm{His}_{6} \mathrm{Er}^{\mathrm{R}}\right)$ & pDPV485 > DPBa89 & This study \\
\hline DPBa218 & АypeB::.pDPV486 (YpeB $\left.{ }^{\mathrm{V} 435 \mathrm{C}}-\mathrm{His}_{6} \mathrm{Er}^{\mathrm{R}}\right)$ & pDPV486 > DPBa89 & This study \\
\hline DPBa219 & АypeB::pDPV487 (YpeB $\left.{ }^{\mathrm{Q} 438 \mathrm{C}}-\mathrm{His}_{6} \mathrm{Er}^{\mathrm{R}}\right)$ & pDPV487 > DPBa89 & This study \\
\hline DPBa220 & АypeB::pDPV488 (YpeB ${ }_{\triangle 25-203}$ K437C-His $\mathrm{Er}^{\mathrm{R}}$ ) & pDPV488 > DPBa89 & This study \\
\hline DPBa221 & pDPV488 $\left(\right.$ YpeB $\left._{\triangle 25-203} \mathrm{~K} 437 \mathrm{C}-\mathrm{His}_{6} \mathrm{Er}^{\mathrm{R}}\right)$ & pDPV488 > 34F2 & This study \\
\hline DPBa222 & АypeB::pDPV489 (YpeB ${ }_{\Delta 25-203}$ S358C-His $\left.\mathrm{Er}^{\mathrm{R}}\right)$ & pDPV489 > DPBa89 & This study \\
\hline DPBa223 & pDPV489 (YpeB ${ }_{\Delta 25-203}$ S358C-His $\left.\mathrm{Er}^{\mathrm{R}}\right)$ & pDPV489 > 34F2 & This study \\
\hline \multicolumn{4}{|l|}{ E.coli } \\
\hline BTH101 & & & Euromedex \\
\hline DPVE545 & pKT25-zip + pUT18C-zip & pKT25-zip + pUT18C-zip > BTH101 & Euromedex \\
\hline DPVE546 & pKT25 + pUT18C & & This study \\
\hline DPVE547 & $p 25-Y p e B_{21-446}+p 18-Y p e B_{21-446}$ & pDPV494 + pDPV490 > BTH101 & This study \\
\hline DPVE548 & p25-YpeB $21-446+p 18-Y p e B_{21-202}$ & pDPV494 + pDPV491 > BTH101 & This study \\
\hline DPVE549 & p25-YpeB $21-446+$ p18-YpeB $203-446$ & pDPV494 + pDPV492 > BTH101 & This study \\
\hline DPVE550 & p25-YpeB $21-446+p 18-S l e B_{125-253}$ & pDPV494 + pDPV493 > BTH101 & This study \\
\hline DPVE551 & p25-YpeB $21-202+p 18-Y p e B_{21-446}$ & pDPV495 + pDPV490 > BTH101 & This study \\
\hline DPVE552 & p25-YpeB $21-202+p 18-Y p e B_{21-202}$ & pDPV495 + pDPV491 > BTH101 & This study \\
\hline DPVE553 & p25-YpeB $21-202+$ p18-YpeB $203-446$ & pDPV495 + pDPV492 > BTH101 & This study \\
\hline DPVE554 & p25-YpeB $21-202+$ p18-SleB $125-253$ & pDPV495 + pDPV493 > BTH101 & This study \\
\hline DPVE555 & p25-YpeB $203-446+p 18-Y_{p e B} 21-446$ & pDPV496 + pDPV490 > BTH101 & This study \\
\hline DPVE556 & $p 25-Y p e B_{203-446}+p 18-Y p e B_{21-202}$ & pDPV496 + pDPV491 > BTH101 & This study \\
\hline DPVE557 & p25-YpeB $203-446+p 18-Y p e B_{203-446}$ & pDPV496 + pDPV492 > BTH101 & This study \\
\hline DPVE558 & p25-YpeB $203-446+p 18-S l e B_{125-253}$ & pDPV496 + pDPV493 > BTH101 & This study \\
\hline DPVE559 & p25-SleB $125-253+$ p18-YpeB $21-446$ & pDPV497 + pDPV490 > BTH101 & This study \\
\hline DPVE560 & p25-SleB $125-253+$ p18-YpeB $21-202$ & pDPV497 + pDPV491 > BTH101 & This study \\
\hline DPVE561 & p25-SleB $125-253+$ p18-YpeB $203-446$ & pDPV497 + pDPV492 > BTH101 & This study \\
\hline DPVE562 & p25-SleB $125-253+$ p18-SleB $125-253$ & pDPV497 + pDPV493 > BTH101 & This study \\
\hline
\end{tabular}

Plasmids

pBKJ236

[27]

pKT25-zip

Euromedex

pUT18C-zip

Euromedex 
Table 1 Bacterial strains and plasmids (Continued)

\begin{tabular}{|c|c|c|c|}
\hline Strain & Genotype & Construction $^{a}$ & Source \\
\hline pKT25 & & & Euromedex \\
\hline pUT18C & & & Euromedex \\
\hline pDPV392 & & pBKJ236:: $\triangle y p e B$ & {$[20]$} \\
\hline pDPV424 & & pBKJ236:: $\triangle$ sleB уре $B_{1-446}$ His $_{6}$ & {$[20]$} \\
\hline pDPV448 & & pBKJ236:: $\triangle$ sleB ype $B_{\triangle 25-203}-\mathrm{His}_{6}$ & {$[20]$} \\
\hline pDPV476 & & 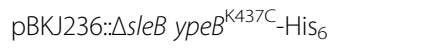 & This study \\
\hline pDPV477 & & pBKJ236:: $\Delta$ sleB ypeB ${ }^{\mathrm{S358C}}-\mathrm{His}_{6}$ & This study \\
\hline pDPV478 & & pBKJ236:: $\Delta$ sleB ypeB ${ }^{\mathrm{T} 328 \mathrm{C}}-\mathrm{His}_{6}$ & This study \\
\hline pDPV479 & & pBKJ236:: $\triangle$ sleB ypeB ${ }^{\mathrm{M} 282 \mathrm{C}}{ }_{-} \mathrm{His}_{6}$ & This study \\
\hline pDPV480 & & pBKJ236:: $\triangle$ sleB ypeB ${ }^{\Upsilon 339 C_{-}}$His $_{6}$ & This study \\
\hline pDPV481 & & pBKJ236:: $\Delta$ sleB ypeB $B^{\mathrm{E} 314 C_{-}} \mathrm{His}_{6}$ & This study \\
\hline pDPV482 & & 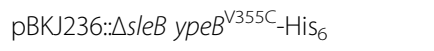 & This study \\
\hline pDPV483 & & 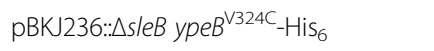 & This study \\
\hline pDPV484 & & pBKJ236:: $\Delta s$ leB ypeB $B^{T 420 C_{-} H_{i}}$ & This study \\
\hline pDPV485 & & pBKJ236:: $\triangle$ sleB ype $B^{\mathrm{A} 324 C_{-}} \mathrm{His}_{6}$ & This study \\
\hline pDPV486 & & pBKJ236:: $\Delta s$ leB ypeB $B^{\mathrm{V} 435 \mathrm{C}_{-} \mathrm{His}_{6}}$ & This study \\
\hline pDPV487 & & pBKJ236:: $\Delta$ sleB ypeB ${ }^{\mathrm{Q} 438 C_{-}} \mathrm{His}_{6}$ & This study \\
\hline pDPV488 & & pBKJ236:: $\Delta s$ leB ypeB $B_{\Delta 25-203}$ K437C-His & This study \\
\hline pDPV489 & & pBKJ236:: $\Delta s$ leB урев ${ }_{\Delta 25-203}$ S358C-His 6 & This study \\
\hline pDPV490 & & pUT18C:::ypeB ${ }_{21-446}$ & This study \\
\hline pDPV491 & & pUT18C:::ypeB $21-202$ & This study \\
\hline pDPV492 & & pUT18C::ypeB $203-446$ & This study \\
\hline pDPV493 & & pUT18C::sleB $B_{125-253}$ & This study \\
\hline pDPV494 & & pKT25::ypeB $21-446$ & This study \\
\hline pDPV495 & & pKT25::ypeB $21-202$ & This study \\
\hline pDPV496 & & pKT25::ypeB $203-446$ & This study \\
\hline pDPV497 & & pKT25::sleB $125-253$ & This study \\
\hline
\end{tabular}

${ }^{a}>$ indicates transformation of the indicated plasmid into the indicated strain

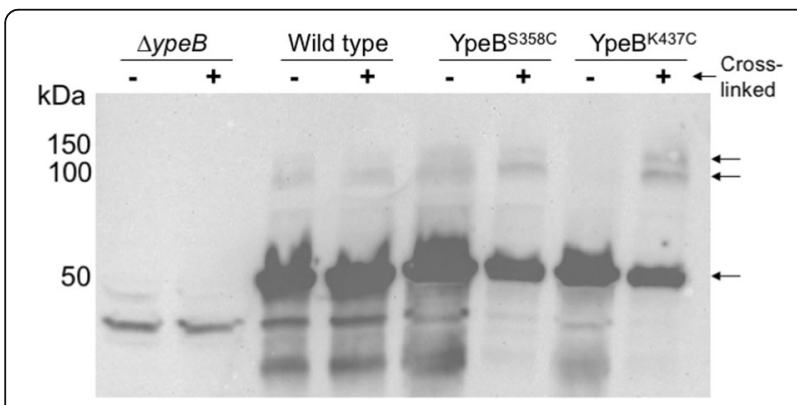

Fig. 1 YpeB-Cys can be cross-linked in spores. Ten optical density units of decoated dormant spores were incubated with $5 \mathrm{mM}$ APB for 30 $\min$ at $37^{\circ} \mathrm{C}$ in reduced light and then irradiated with UV light for 15 min. Cross-linked spores were lyophilized and mechanically broken, and proteins were extracted. Whole spore lysates were then visualized via Western blot using anti-YpeB antibodies [20]. The positions of protein size markers are indicated on the left. The YpeB monomer is indicated by the arrow at $50 \mathrm{kDa}$, higher migrating bands are indicated by arrows at $\sim 100$ and $150 \mathrm{kDa}$ in YpeB-Cys cross-linked samples domain alone does not. However, YpeB-Cys substitutions at some positions in the $\mathrm{C}$-terminal domain can be crosslinked to other YpeB molecules, suggesting close approach of C-terminal domains within the dormant spore, though without a sufficiently strong role in dimerization to be detected using the two-hybrid system. The C-terminal domain alone can interact with fulllength YpeB, suggesting that either both domains are required for stable interaction with the isolated C-terminal domain, or that multimerization of the full-length protein allows further interaction with the isolated C-terminal domain.

YpeB was not demonstrated to form cross-links to SleB from selected residues within the YpeB C-terminal PepSY domains. This may indicate that the YpeB C-terminal domain, although required for stabilization of SleB [20], is not directly interacting with SleB within the dormant spore. It is also possible that these selected 


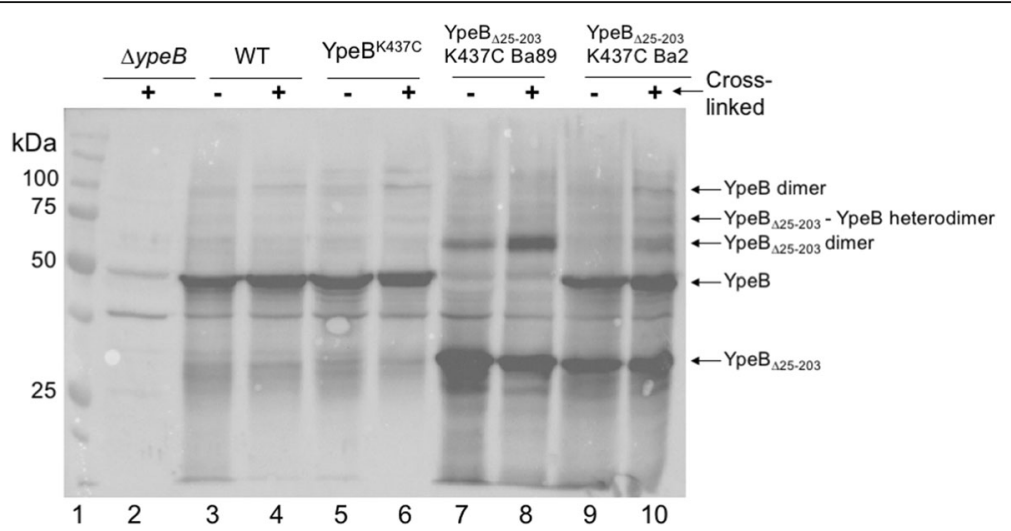

Fig. 2 YpeB ${ }_{\triangle 25-203}$-Cys can be cross-linked in spores. Ten optical density units of decoated dormant spores were cross-linked with APB as described previously. Cross-linked spores were lyophilized and mechanically broken, and proteins were extracted. Whole spore lysates were then visualized via western blot using anti-YpeB antibodies [20]. The positions of protein size markers (lane 1) are indicated on the left. YpeB monomer is indicated by the arrow at $50 \mathrm{kDa}$. $Y_{p e B} \mathrm{~B}_{\triangle 25-203}$ monomer is indicated at roughly $\sim 30 \mathrm{kDa}$. YpeB multimers are visualized in $\mathrm{YpB}^{\mathrm{K}}{ }^{\mathrm{K} 37 \mathrm{C}}$ (lane 6) migrating at 100 and $150 \mathrm{kDa}$. YpeB ${ }_{\triangle 25-203}$ multimers are indicated in $Y_{p e B} B_{\triangle 25-203} \mathrm{~K} 437 \mathrm{C}$ (lane 8) migrating $\sim 60 \mathrm{kDa}$. A putative YpeB-YpeB ${ }_{\triangle 25-203}$ heterodimer (lane 10) is indicated at $\sim 80 \mathrm{kDa}$, in addition to homodimers identified in previous lanes

residues are not in correct orientation to detect an YpeB-SleB interaction. YpeB multimerization may be required for interaction or stabilization of SleB in vivo. Both $\mathrm{N}$ - and C-terminal YpeB domains have been demonstrated to be required for SleB stabilization [20, 21 ], however both domains may be required not because of direct interaction with SleB but rather these domains are necessary for multimer formation. Previous work demonstrated that the YpeB N-terminal can

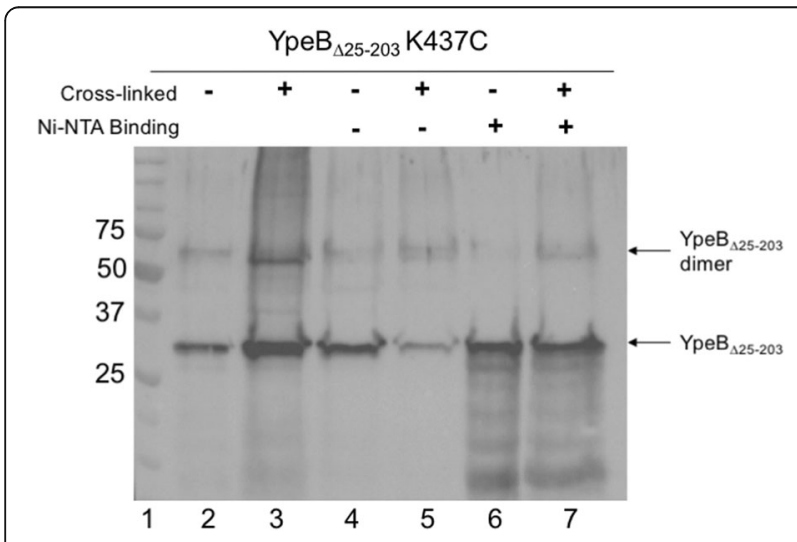

Fig. 3 Column-bound YpeB ${ }_{\triangle 25-203}$ K437C cross-linked complex. Two hundred optical density units of decoated dormant spores were cross-linked with APB. Cross-linked spores were lyophilized and mechanically broken. Proteins were extracted with $8 \mathrm{M}$ urea binding buffer for $2 \mathrm{~h}$. Spore lysates (lanes 2-3) were then passed over a $\mathrm{Ni}^{2+}$ NTA column to isolate $\mathrm{YpeB}_{\triangle 25-203-\mathrm{His}_{6}}$ in addition to those proteins covalently bound via cross-links. Flow-thru (lanes 4-5) and bound (lanes 6-7) fractions were visualized via western blot using anti-YpeB antibodies [20]. The positions of protein size markers (lane 1) are indicated on the left. YpeB $\triangle 25-203 \mathrm{~K} 437 \mathrm{C}$ monomers and dimers were detected in both the load (lane 3) and bound (lane 7) fractions of cross-linked spore samples cause inhibition of SleB activity in vitro [21] and that a region beyond the first PepSY domain was required for stable incorporation of both YpeB and SleB into the spore [20]. Cross-linking data now demonstrates that residues beyond the first PepSY domain appear to be close enough to one another to form a dimer within the dormant spore. Bacterial two-hybrid analysis indicates that both the isolated YpeB N- and Cterminal domains are able to interact with full-length YpeB, thus possibly contributing to a larger multimeric structure, and $\mathrm{N}$-terminal $\mathrm{YpeB}$ appears to strongly associate with itself, suggesting it plays a primary role in multimerization.

The structure of the YpeB C-terminal PepSY domains of B. megaterium has been solved [29]. Authors of the structure suggested a possible binding pocket within a channel traversing the YpeB C-terminal domain, outlined by positive charges of four lysine residues (K345, K347, K361 and K366) [29]. YpeB ${ }^{\mathrm{S} 358 \mathrm{C}}$, one of the residues demonstrated to be reactive in our cross-linking experiments lies directly within this potential bindingpocket. $\mathrm{YpeB}^{\mathrm{K} 437 \mathrm{C}}$, the other reactive residue, lies just beneath the channel but is still surface exposed. It is not immediately obvious how multiple $\mathrm{C}$-terminal domains of YpeB may interact relative to this pocket.

Previous work identified conserved amino acid residues in YpeB PepSY domains required for YpeB, and subsequent SleB, stabilization in dormant spores [20]. In context of the structure of the YpeB PepSY domains, these amino acids were predicted to contribute to stabilization of the structure through intra-molecular interactions [29]. YpeB structure could be a major factor in maintaining stability of any larger multimer in which it could be involved and as such even minor disruption 


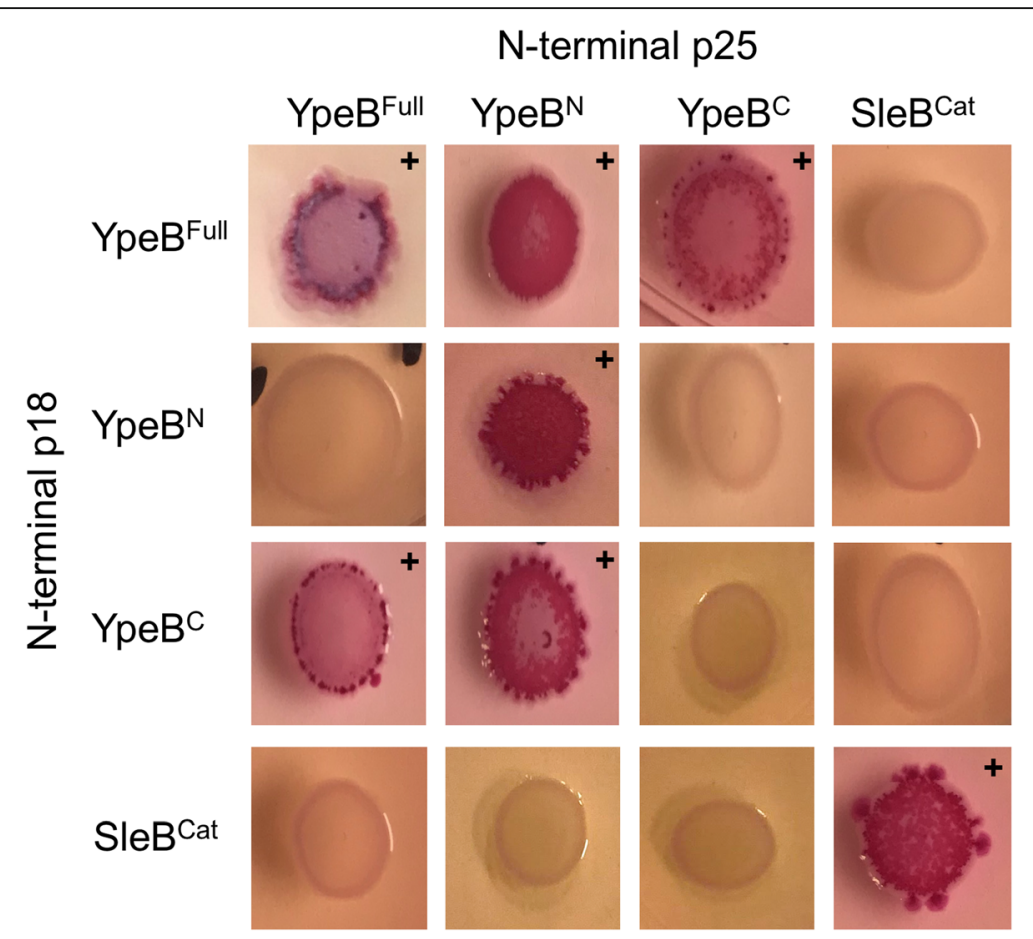

Fig. 4 YpeB and SleB interaction detection by two-hybrid analysis. Individual domains of YpeB and SleB were inserted into both pUT18C and pKT25 creating N-terminal fusions to domains of adenylate cyclase. Plasmids were then co-transformed and screened for protein-protein interactions. Red colonies (+) indicate positive interactions. YpeB ${ }^{N}$ demonstrates strong self-association, while both $Y_{p e B}{ }^{C}$ and $Y_{p e B}{ }^{N}$ appear to interact with YpeB ${ }^{\text {Full }}$. No interactions of YpeB domains with SleB ${ }^{\text {Cat }}$ were detected, but SleB ${ }^{\text {Cat }}$ was found to interact with itself. Images are representative of three biological replicates

of its structure may ultimately lead to degradation during spore formation [20].

Although YpeB cross-linked to SleB was not detected, the list of residues tested was by no means exhaustive and it is entirely possible that the two proteins do interact, especially via the YpeB N-terminal domain [21]. YpeB may also interact with other proteins such as $\mathrm{HtrC}$, which has been previously demonstrated to specifically cleave YpeB during spore germination [30]. Interaction between these, and likely other, proteins on the surface of the dormant spore membrane may serve to stabilize the proteins during long-term dormancy, and to play a key role during spore germination.

\section{Conclusions}

The YpeB protein likely exists as a dimer or higher-order multimer in the dormant spore. Both the $\mathrm{N}$ - and $\mathrm{C}$-terminal YpeB domains contribute to multimerization. SleB likely also exists as an oligomer, and SleB and YpeB may be found together within a protein complex. Disassembly of this complex during spore germination likely allows SleB to become active in spore cortex degradation. Further study of this protein complex may contribute to the development of methods to inhibit or stimulate germination, allowing more effective spore decontamination or more effective use of spores as applied microbial agents.

\section{Methods}

\section{Strain construction}

Site-directed mutagenesis by overlap extension PCR [31] was performed to create cysteine point mutants within $y p e B$. PCR products were then cloned into the $y p e B$ complementation plasmid (pDPV424 [20]) via restriction-free cloning [32]. Plasmids were sequenced to verify cysteine codon substitutions and introduced into $B$. anthracis through conjugation as described previously [20, 27]. Strains with plasmid integrations (Table 1) were selected by shifting the temperature to $42^{\circ} \mathrm{C}$ and verified via PCR as described previously [20].

Construction of strains for two hybrid assays was performed as follows. Desired ypeB and sleB domains were PCR amplified using primers with flanking restriction sites. PCR products were then restriction enzymedigested along with selected vectors pUT18C and pKT25 (Euromedex). Ligations were carried out to insert $y p e B$ or $s l e B$ domain sequences in frame with $\mathrm{N}$-terminally fused p18 or p25 domains of adenylate cyclase. 
Plasmids were then co-transformed into BTH101 (Euromedex) to test potential interactions.

\section{Spore preparation}

B. anthracis spores were prepared in liquid Modified G medium [33] with antibiotics where necessary. Spores were harvested after $3-4$ days incubation at $37^{\circ} \mathrm{C}$ and washed in water for several days until $>95 \%$ free of vegetative cells and cell debris. Decoated spores were prepared as described previously [20]. Briefly, spores were suspended in decoating solution $(50 \mathrm{mM}$ Tris- $\mathrm{HCl}$ $\mathrm{pH} 8,8 \mathrm{M}$ Urea, $1 \%$ SDS, $50 \mathrm{mM}$ dithiothreitol) and incubated for $1 \mathrm{~h}$ at $37^{\circ} \mathrm{C}$. Spores were centrifuged at 8 , $000 \mathrm{x} \mathrm{g}$ for $2 \mathrm{~min}$, and the decoating solution was removed. This procedure was repeated, followed by 5 washes with deionized water.

\section{Cross-linking}

Ten optical density units of decoated spores were suspended in PBS pH 7.5 and APB crosslinker (Sigma) was added to a final concentration of $5 \mathrm{mM}$. Decoated spores were incubated with APB at $37^{\circ} \mathrm{C}$ for $30 \mathrm{~min}$ in the dark. Samples were then exposed to UV light for an additional $15 \mathrm{~min}$ at room temperature. Following UV exposure, cross-linked spores were centrifuged at $10,000 \mathrm{x}$ g for 1 min and the supernatant was removed. Cross-linked spore pellets were stored at $-80^{\circ} \mathrm{C}$ until later use.

\section{Western blotting}

Spores were lyophilized and then were broken mechanically with $100 \mathrm{mg} 0.1 \mathrm{~mm}$ glass beads using Wig-L-Bug bead beaters for 20 pulses of $30 \mathrm{~s}$ each at $4,200 \mathrm{rpm}$. Samples were stored on ice between cycles. Proteins were extracted with $0.125 \mathrm{M}$ Tris- $\mathrm{HCl} \mathrm{pH} 6.8,4 \%$ SDS, $10 \% \beta$-mercaptoethanol, $10 \%$ glycerol, $0.004 \%$ bromophenol blue and run on SDS-polyacrylamide gel electrophoresis. YpeB and SleB were detected via western blot as described previously [20,30]. Briefly, proteins were transferred to Amersham Hybond-P PVDF membranes (GE Healthcare). Anti-YpeB and anti-SleB antibodies were used at 1:3,000 and 1:1,000 dilutions, respectively, and horseradish peroxidase-conjugated secondary goat anti-rabbit antibodies (Bio-Rad) were used at 1:200,000 dilution. Antibody detection utilized chemiluminescence (Clarity Max Western ECL substrate; Bio-Rad).

\section{YpeB-His6 column chromatography}

Following cross-linking of 200 OD units of decoated spores, frozen pellets were lyophilized and broken as described above. Broken spores were suspended in Urea Binding Buffer ( $8 \mathrm{M}$ Urea, $500 \mathrm{mM} \mathrm{NaCl}, 50 \mathrm{mM}$ Tris$\mathrm{HCl}, 30 \mathrm{mM}$ imidazole, $\mathrm{pH} 7.5$ ) and incubated at $4{ }^{\circ} \mathrm{C}$ for $2 \mathrm{~h}$. The samples were centrifuged at $6,800 \mathrm{x}$ g for 10 min, and the soluble fraction was collected, filtered, and loaded onto a $1 \mathrm{~mL}$ Ni Sepharose HisTrap HP (GE Healthcare) column equilibrated in Urea Binding Buffer. Bound YpeB-His6 was eluted with Urea Elution Buffer (8 M Urea, $500 \mathrm{mM} \mathrm{NaCl}, 50 \mathrm{mM}$ Tris- $\mathrm{HCl}, 1 \mathrm{M}$ imidazole, $\mathrm{pH}$ 7.5). Fractions were stored at $-80^{\circ} \mathrm{C}$ for western blot analysis.

\section{Bacterial adenylate cyclase two-hybrid assay}

Protein interactions were screened via spotting $2 \mu \mathrm{l}$ of co-transformed overnight culture on MacConkey agar (ampicillin $100 \mu \mathrm{g} / \mathrm{ml}$, kanamycin $50 \mu \mathrm{g} / \mathrm{ml}, 1 \%$ maltose, $0.5 \mathrm{mM}$ IPTG). Spotted plates were incubated for $48 \mathrm{~h}$ at $30^{\circ} \mathrm{C}$. Positive interactions were visualized by acidification of the media resulting in production of red coloration.

\section{Additional file}

Additional file 1 YpeB-cysteine mutant strain functional screens. Examination of Cys-substituted YpeB protein functionality in germination rate, proteolysis during germination, assembly into the spore, and stabilization of SleB in the spore. (PDF $358 \mathrm{~kb}$ )

\section{Abbreviations}

APB: P-azidophencyl bromide; DPA: Dipicolinic acid; OD: Optical density; PCR: Polymerase chain reaction; SDS: Sodium dodecyl sulfate

\section{Acknowledgements \\ We thank Keane Dye for help in constructing mutant alleles, and Florian Schubot, Jordan Mancl, and Kylie Ryan for contributing E. coli strains and assisting with protein modeling. Some of the data in this manuscript were previously presented in a poster at the 2019 ASM Biothreats conference. We thank the Virginia Tech Open Access Subvention Fund for support of publication costs.}

\section{Authors' contributions}

CVS and DLP designed and interpreted the experiments. CVS completed the experimental work. Both authors wrote and approved the final manuscript.

\section{Funding}

Research reported in this publication was supported by the National Institute of Allergy and Infectious Disease of the National Institutes of Health under award R21Al088298. The funding agency had no role in the design or interpretation of the study or in writing of the manuscript.

\section{Availability of data and materials}

All data generated or analyzed during this study are included in this published article and its supplementary information files.

Ethics approval and consent to participate

Not applicable

Consent for publication

Not applicable

Competing interests

The authors declare that they have no competing interests.

Received: 17 May 2019 Accepted: 18 July 2019

Published online: 26 July 2019

\section{References}

1. Mock M, Fouet A. Anthrax. Annu Rev Microbiol. 2001;55:647-71.

2. Setlow P. Spore germination. Curr Opin Microbiol. 2003;6(6):550-6. 
3. Setlow P. Spores of Bacillus subtilis: their resistance to and killing by radiation, heat and chemicals. J Appl Microbiol. 2006:101(3):514-25.

4. Setlow P. I will survive: DNA protection in bacterial spores. Trends Microbiol. 2007;15(4):172-80

5. Heffron JD, Lambert EA, Sherry N, Popham DL. Contributions of four cortex lytic enzymes to germination of Bacillus anthracis spores. J Bacteriol. 2010; 192(3):763-70

6. Giebel JD, Carr KA, Anderson EC, Hanna PC. The germination-specific lytic enzymes SleB, CWIJ1, and CwIJ2 each contribute to Bacillus anthracis spore germination and virulence. J Bacteriol. 2009;191(18):5569-76.

7. Heffron JD, Orsburn B, Popham DL. Roles of germination-specific lytic enzymes CWIJ and SleB in Bacillus anthracis. J Bacteriol. 2009;191(7):2237-47.

8. Atrih A, Zollner P, Allmaier G, Foster SJ. Structural analysis of Bacillus subtilis 168 endospore peptidoglycan and its role during differentiation. J Bacteriol. 1996:178(21):6173-83.

9. Chen Y, Fukuoka S, Makino S. A novel spore peptidoglycan hydrolase of Bacillus cereus: biochemical characterization and nucleotide sequence of the corresponding gene, sleL. J Bacteriol. 2000;182(6):1499-506.

10. Heffron JD, Sherry N, Popham DL. In vitro studies of peptidoglycan binding and hydrolysis by the Bacillus anthracis germination-specific lytic enzyme SleB. J Bacteriol. 2011;193(1):125-31.

11. Lambert EA, Sherry N, Popham DL. In vitro and in vivo analyses of the Bacillus anthracis spore cortex Iytic protein SleL. Microbiology. 2012; 158(Pt 5):1359-68.

12. Makino S, Moriyama R. Hydrolysis of cortex peptidoglycan during bacterial spore germination. Med Sci Monit. 2002;8(6):RA119-27.

13. Popham DL, Helin J, Costello CE, Setlow P. Muramic lactam in peptidoglycan of Bacillus subtilis spores is required for spore outgrowth but not for spore dehydration or heat resistance. Proc Natl Acad Sci U S A. 1996;93(26):15405-10.

14. Meador-Parton J, Popham DL. Structural analysis of Bacillus subtilis spore peptidoglycan during sporulation. J Bacteriol. 2000;182:4491-9.

15. Warth AD, Strominger JL. Structure of the peptidoglycan of bacterial spores: occurrence of the lactam of muramic acid. Proc Natl Acad Sci U S A. 1969: 64(2):528-35.

16. Bagyan I, Setlow P. Localization of the cortex lytic enzyme CwlJ in spores of Bacillus subtilis. J Bacteriol. 2002;184(4):1219-24.

17. Chirakkal H, O'Rourke M, Atrih A, Foster SJ, Moir A. Analysis of spore cortex lytic enzymes and related proteins in Bacillus subtilis endospore germination. Microbiology. 2002;148(Pt 8):2383-92.

18. Popham DL, Bernhards CB. Spore peptidoglycan. Microbiol Spectrum. 2015; 3(6). doi: https://doi.org/10.1128/microbiolspec.TBS-0005-2012.

19. Moriyama R, Fukuoka H, Miyata S, Kudoh S, Hattori A, Kozuka S, Yasuda Y, Tochikubo K, Makino S. Expression of a germination-specific amidase, SleB, of bacilli in the forespore compartment of sporulating cells and its localization on the exterior side of the cortex in dormant spores. J Bacteriol. 1999;181(8):2373-8.

20. Bernhards CB, Popham DL. Role of YpeB in cortex hydrolysis during germination of Bacillus anthracis spores. J Bacteriol. 2014;196(19):3399-409.

21. Li Y, Butzin XY, Davis A, Setlow B, Korza G, Ustok Fl, Christie G, Setlow P, Hao B. Activity and regulation of various forms of CWlJ, SleB, and YpeB proteins in degrading cortex peptidoglycan of spores of Bacillus species in vitro and during spore germination. J Bacteriol. 2013;195(11):2530-40.

22. Boland FM, Atrih A, Chirakkal H, Foster SJ, Moir A. Complete spore-cortex hydrolysis during germination of Bacillus subtilis 168 requires SleB and YpeB. Microbiology. 2000;146(Pt 1):57-64.

23. Moriyama R, Kudoh S, Miyata S, Nonobe S, Hattori A, Makino S. A germination-specific spore cortex-lytic enzyme from Bacillus cereus spores: cloning and sequencing of the gene and molecular characterization of the enzyme. J Bacteriol. 1996;178(17):5330-2.

24. Hu K, Yang H, Liu G, Tan H. Cloning and identification of a gene encoding spore cortex-lytic enzyme in Bacillus thuringiensis. Curr Microbiol. 2007;54(4):292-5.

25. Moriyama R, Hattori A, Miyata S, Kudoh S, Makino S. A gene (sleB) encoding a spore cortex-lytic enzyme from Bacillus subtilis and response of the enzyme to L-alanine-mediated germination. J Bacteriol. 1996;178(20):6059-63.

26. Gao X, Wang J, Yu DQ, Bian F, Xie BB, Chen XL, Zhou BC, Lai LH, Wang ZX, Wu JW, et al. Structural basis for the autoprocessing of zinc metalloproteases in the thermolysin family. Proc Natl Acad Sci U S A. 2010; 107(41):17569-74
27. Janes BK, Stibitz S. Routine markerless gene replacement in Bacillus anthracis. Infect Immun. 2006;74(3):1949-53.

28. Jing $X$, Robinson HR, Heffron JD, Popham DL, Schubot FD. The catalytic domain of the germination-specific lytic transglycosylase SleB from Bacillus anthracis displays a unique active site topology. Proteins. 2012; 80(10):2469-75

29. Ustok Fl, Chirgadze DY, Christie G. Crystal structure of the PepSY-containing domain of the YpeB protein involved in germination of Bacillus spores. Proteins. 2015;83(10):1914-21.

30. Bernhards CB, Chen Y, Toutkoushian H, Popham DL. HtrC is involved in proteolysis of YpeB during germination of Bacillus anthracis and Bacillus subtilis spores. J Bacteriol. 2015;197(2):326-36.

31. Ho SN, Hunt HD, Horton RM, Pullen JK, Pease LR. Site-directed mutagenesis by overlap extension using the polymerase chain reaction. Gene. 1989;77(1):51-9.

32. van den Ent F, Lowe J. RF cloning: a restriction-free method for inserting target genes into plasmids. J Biochem Biophys Methods. 2006;67(1):67-74

33. Kim HU, Goepfert JM. A sporulation medium for Bacillus anthracis. J Appl Bacteriol. 1974:37(2):265-7.

\section{Publisher's Note}

Springer Nature remains neutral with regard to jurisdictional claims in published maps and institutional affiliations.
Ready to submit your research? Choose BMC and benefit from:

- fast, convenient online submission

- thorough peer review by experienced researchers in your field

- rapid publication on acceptance

- support for research data, including large and complex data types

- gold Open Access which fosters wider collaboration and increased citations

- maximum visibility for your research: over $100 \mathrm{M}$ website views per year

At $\mathrm{BMC}$, research is always in progress.

Learn more biomedcentral.com/submissions 